Research Article

\title{
Generalized Thermopiezoelectricity with Memory-Dependent Derivative and Transient Thermoelectromechanical Responses Analysis
}

\author{
Jianzhong Sun (iD) and Yaping Wu \\ School of Civil Engineering, Lanzhou Jiaotong University, Lanzhou 730070, Gansu, China \\ Correspondence should be addressed to Jianzhong Sun; sunjzh@mail.lzjtu.cn
}

Received 19 June 2020; Revised 10 November 2020; Accepted 1 February 2021; Published 22 February 2021

Academic Editor: Jinyang $\mathrm{Xu}$

Copyright (C) 2021 Jianzhong Sun and Yaping Wu. This is an open access article distributed under the Creative Commons Attribution License, which permits unrestricted use, distribution, and reproduction in any medium, provided the original work is properly cited.

\begin{abstract}
In this paper, a novel generalized thermoelastopiezoelectric model is established by introducing memory-dependent derivative, which might be superior to fractional ones: the form is unique, while the fractional-order theories have various expressions with different authors; it is more intuitionistic for reflecting the memory effect; the physical meanings of the related memorydependent differential equations are more clear, which are determined by the essence of their definitions; the time-delay and kernel function can be chosen freely based on the necessity of practical applications. The newly constructed model is applied to the transient shock analysis for piezoelectric medium under heating loads. Laplace transformation techniques are employed to solve the governing equations. In numerical implementation, the problem of a semi-infinite piezoelectric medium is considered under the two different cases. The transient responses, that is, temperature, displacement, stress, and electric potential, are illustrated graphically. The parametric studies are performed to analyze the effects of time-delay and kernel function on the transient thermoelastopiezoelectric responses. This work may provide a new approach to explore the transient responses' behavior for piezoelectric materials serving in nonisothermal environment.
\end{abstract}

\section{Introduction}

The piezoelectric materials serve as a new class of smart structure, which have been extensively applied for their excellent performance in sensors and actuators. It is noted that a critical review article that focused on the piezoelectric material's applications in the disturbance sensing and control of intelligent structures has been reported [1]. Nevertheless, in most engineering cases, the structures composed of piezoelectric materials commonly serve in the nonisothermal environment. In such situations, the pyroelectric effect in the analysis of thermoelectromechanical behavior is imperative to be considered seriously. Actually, the strain field, electric field, and temperature field in real piezoelectric ceramics are always closely coupled simultaneously [2-4]. Thus, the interaction mechanism of thermoelastopiezoelectric coupling fields in piezoelectric ceramics should be given a comprehensive understanding. When suffering from heating loads, thermal stresses in piezoelectric materials are excessively important. Considering the thermal/electric induced deformation in piezoelectric medium, the coupled thermopiezoelectricity theory was proposed [5] and the related physical laws were completed [6]. So far, a series of practical problems have been investigated: transient analysis [7], vibration control [8], cracking problems [9], wave propagation [10], and so forth.

However, the classical thermopiezoelectricity [5] predicts an infinite speed of thermal propagation, which evidently contradicts the physical facts [11-13]. The introduction of conventional Fourier's heat conduction law in [5] leads to such limitation. To remove this defect, the nonclassical heat conduction models have been extended. The first of such models is commonly referred to as $\mathrm{C}-\mathrm{V}$ equation [14, 15]. Then, the paradox in [5] has been 
eliminated by establishing a generalized thermopiezoelectricity theory [16], which involves a finite speed of thermal wave. Based on this theory, some interesting works have been fulfilled: two-dimensional shock problem for a thick piezoelectric plate $[17,18]$, the coupling problem for a structure composed of temperature-dependent materials [19], dynamic responses of a piezoelectric rod [20], transient responses for a one-dimensional functionally graded piezoelectric medium [21], the reflection and refraction of longitudinal waves in piezoelectric materials [22], and so forth.

Note that the heating loads in these problems share the following features: high flux, micro-/nanoscale, and sudden shocking. In such complex situations, the heat flux at a point is commonly interrelated to the history of heat carriers reaching the point at a given time, which can be viewed as a history-dependent process. Therefore, the generalized heat conduction models, such as damped wave model [14, 15], parabolic two-step model $[23,24]$, and hyperbolic two-step model [25], may not be accurate in the predictions of temperature distributions when investigating the energy transfer for transient heating problems at micro-/nanoscale [26]. For the detailed report of heat conduction laws, the reader is referred to the review article in [27].
It is well known that the complicated real world always exhibits the power-law characteristic. One may conclude that the integer-order derivative models and even nonlinear models may fail in such cases. To accurately depict the power-law phenomenon (e.g., nonlocal effect and historyand path-dependency), the introduction of new mathematical modeling method, like fractional calculus, is very necessary. In the latest decades, the increasing applications of fractional derivatives and integrals can be found in various domains of electrical engineering, laser cooling, signal/image processing, control system, and so forth. Particularly, in the researches of heat conduction behavior, it is noteworthy that the introduction of fractional-order derivatives/integrals has been experimentally verified to be a more accurate approach than the classical one [28, 29]. As a powerful mathematical tool, the methodology of fractional calculus has been widely employed in micro-/nanoscale heating problems [30-33]. Inspired by fractional calculus's resounding success, the generalized thermopiezoelectricity has been further extended into fractional ones [34-36]. Generally speaking, fractional calculus contains various types of definitions, the most widely adopted ones of which are Riemann-Liouville and Caputo fractional derivatives.

(i) For Riemann-Liouville definition,

$$
\begin{aligned}
{ }_{a}^{R L} D_{t}^{\alpha} f(t) & =\left(\frac{\mathrm{d}}{\mathrm{d} t}\right)^{n} I^{(n-\alpha)} f(t) \\
& =\frac{1}{\Gamma(n-\alpha)}\left(\frac{\mathrm{d}}{\mathrm{d} t}\right)^{n} \int_{a}^{t} \frac{f(p)}{(t-p)^{\alpha-n+1}} \mathrm{~d} p \quad(n-1 \leq \alpha<n, \quad t>a) .
\end{aligned}
$$

(ii)For Caputo definition,

$$
\begin{aligned}
{ }_{a}^{C} D_{t}^{\alpha} f(t) & =I^{(n-\alpha)} f^{(n)}(t) \\
& =\frac{1}{\Gamma(n-\alpha)} \int_{a}^{t} \frac{f(p)}{(t-p)^{\alpha-n+1}} \mathrm{~d} p, \quad(n-1 \leq \alpha<n, \quad t>a)
\end{aligned}
$$

where $\Gamma(n)=(n-1)$ ! denotes the Gamma function. Obviously, the sequence of fractional integral and integer-order derivative is distinct in the two definitions (1) and (2), which may result in the huge differences in practical applications: the initial conditions for conventional differential equations are not valid for Riemann-Liouville type fractional differential equations, while they are applicable for Caputo type fractional differential equations. More details can be found in the monograph in [37]. It is noteworthy that definition (2) can be further rewritten as

$$
D_{a}^{\alpha} f(t)=\int_{a}^{t} K_{\alpha}(t, p) f^{(n)}(p) \mathrm{d} p, \quad K_{\alpha}(t, p)=\frac{(t-p)^{n-\alpha-1}}{\Gamma(n-\alpha)} .
$$

It is clear that these theories [34-36] may be totally referred to as time-fractional-order generalized thermopiezoelectricity, which can be applied to depict memorydependent behaviors. Nevertheless, it is easily seen that different formulas are proposed by different authors; that is, the aforementioned fractional-order generalized thermopiezoelectricity theories are not unique in the form. Hence, it can be deduced that there has been a widespread debate on this topic and more investigation efforts should be devoted.

Recently, a novel concept of memory-dependent derivative was proposed [38]:

$$
D_{\omega}^{m} f(t)=\int_{t-\omega}^{t} K(t, p) f^{(m)}(p) \mathrm{d} p \quad(\omega>0),
$$


where the kernel function $K(t, p)$ and time-delay $\omega$ can be selected freely. This kind of definition (4) can reflect the memory effect on the delayed interval $[t-\omega, t]$, which varies along with time. The kernel function $K(t, p)$ can be interpreted as the degree of the past effect on the present; thus, the forms $K(t, p)=(1 / \omega)[(p-t / \omega)+1]^{b}(b \geq 0)$ may be more practical. More importantly, as discussed in the article in [38], definition (3) is limited in two aspects: firstly, for a given real number $\alpha$, the kernel $K_{\alpha}(t, p)$ is a fixed function; secondly, it may be invalid in describing the memory effect for larger time $t$ due to the fractional derivative defined on the interval $[a, t]$ with a fixed real number $a$. From the viewpoint of application, the new consideration of memory-dependent derivative may be more rational in describing the memory effect than fractional ones. Firstly, memory effects should be considered with different forms of kernels; that is, the kernel functions can be chosen freely for different physical processes. Secondly, the memory effect of a real process basically occurs in a segment of time, that is, the delayed interval $[t-\omega, t]$.

As the study in [38] made reference to the fact that this kind of definition (4) is better than the fractional one for reflecting the memory effect (instantaneous change rate depends on the past state), it is worth stressing that numerical experiments have been conducted [38] for the linear initial value problems, which show the advantages of memory-dependent derivative in reflecting memory effect. It can be concluded that the new definition (4) is more intuitionistic for understanding the physical meanings and the related memory-dependent differential equation is more expressive. Nowadays, fractionalorder derivative has been used in the thermomechanical response analysis of different material systems [39-43].

Seeing the superiorities of memory-dependent derivative in the aspect of reflecting the memory effect, it can be applied in the dynamic response analysis for piezoelectric materials under heating loads (even micro-/nanoscale heating). The present work is devoted to establishing a novel generalized thermopiezoelectric model by introducing the memorydependent derivative and investigating the transient thermoelastopiezoelectric responses' behavior. Laplace transformation techniques are employed to solve the governing equations. As numerical example, the problem of a semi-infinite piezoelectric medium is considered under the two cases. The transient responses, that is, temperature, displacement, stress, and electric potential, are obtained and illustrated graphically. The effects of time-delay and kernel function on the transient responses are analyzed and discussed in detail. Finally, some conclusions are summarized.

\section{Basic Equations}

For linear piezoelectric medium, the governing equations of generalized thermopiezoelectricity with memory-dependent derivative consist of (in the absence of electric current and free charge) the following:

(i) Motion equation

$$
\sigma_{i j, j}+f_{i}=\rho u_{i, t t},
$$

where $\rho$ is the mass density, $\sigma_{i j}$ is the stress tensor, $u_{i}$ is the displacement vector, and $f_{i}$ is the body force.

(ii) The equation for energy conservation

$$
\rho T_{0} S_{, t}=-q_{i, i}+\rho R,
$$

where $T_{0}, S, q_{i}$, and $R$ are, respectively, the reference temperature, entropy density, heat flux, and heat source intensity.

(iii) Gauss equation and electric field relation

$$
\begin{aligned}
D_{i . i} & =0, \\
E_{i} & =-\phi_{, i},
\end{aligned}
$$

where $D_{i}, E_{i}$, and $\phi$ denote the electric displacement, electric intensity, and electric potential, respectively.

(iv) The kinematic relation

$$
\varepsilon_{i j}=\frac{1}{2}\left(u_{i, j}+u_{j, i}\right)
$$

where $\varepsilon_{i j}$ is the strain tensor.

(v) Constitutive equations

$$
\begin{gathered}
\sigma_{i j}=c_{i j k l} \varepsilon_{k l}-e_{i j k} E_{k}-\beta_{i j} \theta, \\
\rho S=\frac{\rho c_{E}}{T_{0}} \theta+\beta_{i j} \varepsilon_{i j}+p_{i} E_{i}, \\
D_{i}=e_{i j k} \varepsilon_{j k}+d_{i j} E_{j}+p_{i} \theta,
\end{gathered}
$$

where $c_{E}, \theta, c_{i j k l}, e_{i j k}, \beta_{i j}, d_{i j}$, and $p_{i}$ are, respectively, the specific heat, the relative temperature, elastic constants, piezoelectric constant, thermal moduli, dielectric constants, and pyroelectric constant.

(vi) Energy equation

The heat conduction with memory-dependent derivative can be written as

$$
q_{i}+\omega D_{\omega} q_{i}=-\kappa_{i j} \theta_{, j}
$$

Then, combining equations (6), (7a) and (7b), (10), and (12), one can obtain

$$
\left(1+\omega D_{\omega}\right)\left(\rho c_{E} \theta_{, t}+T_{0} \beta_{i j} \varepsilon_{i j, t}+T_{0} p_{i} \phi_{, i t}-\rho R\right)=\kappa_{i j} \theta_{i, j},
$$

where $\kappa_{i j}$ represents the coefficients of thermal conductivity. In addition, $D_{t_{d}}$ is the first order of memory-dependent derivative (4), which has the following form:

$$
D_{\omega} f=D_{\omega}^{1} f=\int_{t-\omega}^{t} K(t, p) f^{(1)}(p) \mathrm{d} p .
$$


In order to properly formulate a problem, appropriate boundary conditions associated with the above governing equations must be adopted. When the displacement, temperature, and electrical potential are prescribed on the surfaces $A_{u}, A_{\theta}$, and $A_{\phi}$, respectively, one has

$$
\begin{aligned}
u_{i} & =\widetilde{u}_{i} \text { on } A_{u}, \\
\theta & =\widetilde{\theta} \text { on } A_{\theta}, \\
\phi & =\widetilde{\phi} \text { on } A_{\phi},
\end{aligned}
$$

where $\widetilde{u}_{i}, \widetilde{\theta}$, and $\widetilde{\phi}$ are the prescribed values. On the other hand, if surface traction, surface flux, and surface charges are separately applied to the surfaces $A_{\sigma}, A_{q}$, and $A_{D}$, the following boundary conditions must be satisfied:

$$
\begin{aligned}
\sigma_{i j} n_{j} & =\widetilde{F}_{i} \text { on } A_{\sigma}, \\
q_{i} n_{i} & =\widetilde{Q} \text { on } A_{q}, \\
D_{i} n_{i} & =\widetilde{d} \text { on } A_{D},
\end{aligned}
$$

where $\widetilde{F}_{i}, \widetilde{Q}$, and $\widetilde{d}$ are the given surface traction, surface flux, and surface charges, respectively. Thus far, the new generalized thermopiezoelectricity model with memorydependent derivative is elaborated.

\section{Transient Thermoelastopiezoelectric Responses for a Semi-Infinite Piezoelectric Medium}

We shall consider a semi-infinite problem of piezoelectric medium, which is assumed to be unstrained and unstressed initially. The polarization direction is parallel to $z$-axis. Since the dimension in one direction ( $z$-axis) is much larger than that in the other directions, the problem can be viewed as a one-dimensional problem. During the analysis, it is further assumed that neither elastic wave nor thermal wave reaches the other end $z=l$, which can be mathematically expressed as $u(l, t) \longrightarrow 0, \theta(l, t) \longrightarrow 0, \phi(l, t) \longrightarrow 0$. From the physics of the problem, it is clear that all the functions are considered to be dependent on $z$ and $t$ only. Thus, equations (5)-(11) are reduced to

$$
\begin{aligned}
\sigma_{z z, z} & =\rho w_{t t}, \\
\rho T_{0} S_{, t}+q_{z, z} & =0, \\
D_{z . z} & =0, \\
E_{z} & =-\phi_{, z}, \\
\varepsilon_{z z} & =w_{, z} \\
\sigma_{z z} & =c_{33} \varepsilon_{z z}-e_{33} E_{z}-\beta_{3} \theta \\
\rho S & =\frac{\rho c_{E}}{T_{0}} \theta+\beta_{3} \varepsilon_{z z}+p_{3} E_{z} \\
D_{z} & =e_{33} \varepsilon_{z z}+d_{3} E_{z}+p_{3} \theta \\
q_{z}+\omega D_{\omega} q_{z} & =-\kappa_{3} \theta_{, z} .
\end{aligned}
$$

By using equations (18b) and (19), (20)-(23), (17) and (18a) will be reduced to the governing differential equations of the current problem:

$$
c_{33} w_{, z z}+e_{33} \phi_{, z z}-\beta_{3} \theta_{, z}=\rho w_{, t t},
$$

$$
e_{33} w_{, z z}-d_{3} \phi_{, z z}+p_{3} \theta_{, z}=0
$$

$\kappa_{3} \theta_{, z z}-\left(1+\omega D_{\omega}\right)\left(\rho c_{E} \theta_{, t}+\beta_{3} T_{0} w_{, z t}-p_{3} T_{0} \phi_{, z t}\right)=0$.

For convenience, the following dimensionless quantities are introduced:

$$
\begin{aligned}
\left(z^{*}, w^{*}, \phi^{*}\right) & =c_{0} \eta_{0}(z, w, \phi), \\
\left(t^{*}, t_{d}^{*}, \tau^{*}\right) & =c_{0}^{2} \eta_{0}\left(t, t_{d}, \tau\right) \\
\sigma_{z z}^{*} & =\frac{\sigma_{z z}}{c_{33}}, \\
\theta^{*} & =\frac{\theta}{T_{0}}, \\
D_{z}^{*} & =\frac{D_{z}}{e_{33}}, \\
c_{0} & =\sqrt{\frac{c_{33}}{\rho}} \\
\eta_{0} & =\frac{\rho c_{E}}{\kappa_{3}} .
\end{aligned}
$$

Employing (27), equations (24)-(26) can be written as follows (the asterisks of dimensionless quantities have been left out for brevity):

$$
\begin{array}{r}
w_{, z z}+g_{1} \phi_{, z z}-g_{2} \theta_{, z}-w_{, t t}=0, \\
w_{, z z}-g_{3} \phi_{, z z}+f_{1} \theta_{, z}=0, \\
\theta_{, z z}-\left(1+\omega D_{\omega}\right)\left(\theta_{, t}+f_{2} w_{, z t}-f_{3} \phi_{, z t}\right)=0,
\end{array}
$$

where

$$
\begin{aligned}
& g_{1}=\frac{e_{33}}{c_{33}}, \\
& g_{2}=\frac{\beta_{3} T_{0}}{c_{33}}, \\
& g_{3}=\frac{d_{3}}{e_{33}}, \\
& f_{1}=\frac{p_{3} T_{0}}{e_{33}}, \\
& f_{2}=\frac{\beta_{3}}{\rho c_{E}}, \\
& f_{3}=\frac{p_{3}}{\rho c_{E}} .
\end{aligned}
$$

Applying the Laplace transform, defined by the relation 


$$
\bar{f}(x, s)=L(f(x, t))=\int_{0}^{\infty} f(x, t) e^{-s t} \mathrm{~d} t,
$$

on both sides of equations (28)-(30), with the prescribed initial conditions, $u(x, 0)=\dot{u}(x, 0)=\theta$ $(x, 0)=\dot{\theta}(x, 0)=\phi(x, 0)=\dot{\phi}(x, 0)=0$, one can obtain

$$
\begin{array}{r}
\bar{w}_{, z z}-s^{2} \bar{w}+g_{1} \bar{\phi}_{, z z}-g_{2} \bar{\theta}_{, z}=0, \\
\bar{w}_{, z z}-g_{3} \bar{\phi}_{, z z}+f_{1} \bar{\theta}_{, z}=0, \\
\bar{\theta}_{, z z}-s_{0}\left(\bar{\theta}+f_{2} \bar{w}_{, z}-f_{3} \bar{\phi}_{, z}\right)=0,
\end{array}
$$

where $s_{0}$ denotes the following cases:

(1) $b=0$; that is, $K(t, p)=1 \quad$ and $s_{0}=s\left[\omega\left(1-e^{-s \omega}\right)+\omega\right]$

(2) $b=1$; that is, $K(t, p)=[(p-t / \omega)+1]$ and $s_{0}=s\left\{2 \omega\left[1-(1 / s \omega)\left(1-e^{-s \omega}\right)\right]+\omega\right]$
(3) $b=2$; that is, $K(t, p)=[(p-t / \omega)+1]^{2}$ and $s_{0}=s\left\{3 \omega\left[(1-(2 / s \omega))+\left(2 / s^{2} \omega^{2}\right)\left(1-e^{-s \omega}\right)\right]\right]$

By eliminating $\bar{\theta}_{, z}$ between equations (33) and (34), we obtain the following equation for $\bar{w}$ and $\bar{\phi}$ :

$$
\left(1+\frac{g_{2}}{f_{1}}\right) \bar{w}_{, z z}-s^{2} \bar{w}+\left(g_{1}-\frac{g_{2} g_{3}}{f_{1}}\right) \bar{\phi}_{, z z}=0 .
$$

After solving for $\bar{\theta}_{, z}$, employing equation (33) and using its derivative $\left(\bar{\theta}_{, z z}\right)$ and equation (35), we can find that $\bar{\theta}$ has the following form:

$$
\bar{\theta}=\frac{1}{g_{2} s_{1}} \bar{w}_{, z z z}-\left(f_{2}+\frac{s^{2}}{g_{2} s_{1}}\right) \bar{w}_{, z}+\frac{g_{1}}{g_{2} s_{1}} \bar{\phi}_{, z z z}+f_{3} \bar{\phi}_{, z}
$$

Using the above equations, we can eliminate $\bar{\theta}$ in (34). Thus, one can obtain

$$
\frac{f_{1}}{g_{2} s_{1}} \bar{w}_{, z z z z}+\left[1-f_{1}\left(f_{2}+\frac{s^{2}}{g_{2} s_{1}}\right)\right] \bar{w}_{, z z}+\frac{f_{1} g_{1}}{g_{2} s_{1}} \bar{\phi}_{, z z z z}+\left(f_{1} f_{3}-g_{3}\right) \bar{\phi}_{, z z}=0 .
$$

Similarly, $\bar{\phi}$ can be obtained between equations (36) and

$$
p \bar{w}_{, z z z z}+q \bar{w}_{, z z}+r \bar{w}=0
$$
(38). Then, we can obtain the final differential equation of $\bar{w}$ as follows:

where

$$
\begin{aligned}
& p=\frac{f_{1}}{g_{2} s_{1}}\left(1-g_{1} \frac{f_{1}+g_{2}}{f_{1} g_{1}-g_{2} g_{3}}\right) \\
& q=1-f_{1}\left(f_{2}+\frac{s^{2}}{g_{2} s_{1}}\right)+\frac{f_{1}^{2} s^{2} g_{1}}{g_{2} s_{1}\left(f_{1} g_{1}-g_{2} g_{3}\right)}-\frac{\left(f_{1} f_{3}-g_{3}\right)\left(f_{1}+g_{2}\right)}{f_{1} g_{1}-g_{2} g_{3}} \\
& r=\left(f_{1} f_{3}-g_{3}\right) \frac{f_{1} s^{2}}{f_{1} g_{1}-g_{2} g_{3}} .
\end{aligned}
$$

The solution of equation (39) can be expressed as

$$
\bar{w}=\sum_{i=1}^{2} \bar{w}_{i} e^{-k_{i} z}
$$

where $k_{i}=\sqrt{\left(-q \pm \sqrt{q^{2}-4 p r}\right) / 2 p}(i=1,2)$ are corresponding characteristic roots of equation (39). Substituting equation (41) into (34) and (36), we can find $\bar{\phi}$ and $\bar{\theta}$, respectively, as follows:

$$
\begin{gathered}
\bar{\phi}=\sum_{i=1}^{2} \bar{\phi}_{i} \bar{w}_{i} e^{-k_{i} z}, \\
\bar{\theta}=\sum_{i=1}^{2} \bar{\theta}_{i} \bar{w}_{i} e^{-k_{i} z},
\end{gathered}
$$

where

$$
\begin{aligned}
& \bar{\phi}_{i}=\frac{1}{f_{1} g_{1}-g_{2} g_{3}}\left[\frac{f_{1} s^{2}}{k_{i}^{2}}-\left(f_{1}+g_{2}\right)\right], \\
& \bar{\theta}_{i}=\frac{k_{i}\left(1-g_{3} \bar{\phi}_{i}\right)}{f_{1}}
\end{aligned}
$$

Introducing the solutions of the displacement and temperature into the dimensionless constitutive equation (20) in Laplace domain,

$$
\bar{\sigma}=\sum_{i=1}^{2} \bar{\sigma}_{i} \bar{w}_{i} e^{-k_{i} z}
$$

where

$$
\bar{\sigma}_{i}=-\left[k_{i}\left(1+g_{1} \bar{\phi}_{i}\right)+g_{2} \bar{\theta}_{i}\right]
$$


To obtain the transient solutions in time domain, an algorithm NILT [44] based on fast Fourier transformation is adopted.

\section{Applications}

Problem 1. The problem considered is that a semi-infinite piezoelectric medium is assumed to be traction-free at one end $(z=0)$ with a transient thermal loading (see Figure 1).

Thermal boundary condition:

A transient thermal loading is applied to the boundary plane $z=0$ in the following form:

$$
\begin{array}{r}
\theta(0, t)=h(t), \\
\text { or } \\
\bar{\theta}(0, s)=\bar{h}(s),
\end{array}
$$

where

$$
\begin{aligned}
& h(t)=H(t), \\
& \text { or } \\
& \bar{h}(s)=\frac{1}{s} .
\end{aligned}
$$

Mechanical boundary condition:

The bounding plane $z=0$ is taken to be traction-free; that is,

$$
\bar{\sigma}(0, t)=0 \text {, or } \bar{\sigma}(0, s)=0 \text {, }
$$

Problem 2. A semi-infinite piezoelectric medium with one end subjected to ramp-type heating loads is investigated (see Figure 1).

Thermal boundary condition:

Ramp-type heating loads are applied to the boundary plane $z=0$ in the following form:

$$
\begin{gathered}
\theta(0, t)=g(t), \\
\text { or } \\
\bar{g}(0, s)=\bar{g}(s)
\end{gathered}
$$

where

$$
g(t)= \begin{cases}0, & t \leq 0 \\ \theta_{1} \frac{t}{t_{0}}, & 0<t \leq t_{0} \\ \theta_{1}, & t>t_{0} .\end{cases}
$$

or

$$
\bar{g}(s)=\frac{\theta_{1}\left(1-e^{-t_{0} s}\right)}{t_{0} s^{2}} .
$$

Mechanical boundary condition:

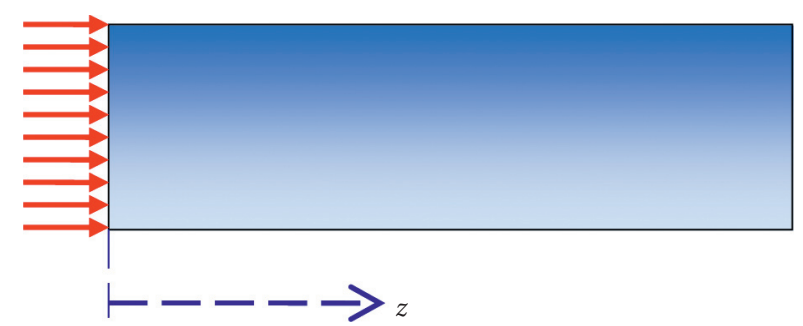

Figure 1: A semi-infinite piezoelectric medium with one end subjected to thermal loading.

The bounding plane $z=0$ has a constant displacement; that is,

$$
w^{\prime}(0, t)=0, \text { or } \overline{w^{\prime}}(0, s)=0
$$

To determine the parameters $\bar{w}_{i}(i=1,2)$ in Problem 1 and Problem 2, boundary conditions (46)-(51) will be employed, and then the solutions in Laplace transform domain are obtained.

\section{Results and Discussion}

The cadmium selenide material is selected for simulation, whose physical material constants are shown in Table 1.

As discussed in [45], if the thermal relaxation time (i.e., $\tau$ ) and time-delay parameter (i.e., $\omega$ ) approach being infinitesimal, the equation of memory-dependent heat conduction model and fractional heat conduction model is equivalent in theory, that is, degenerating into the $\mathrm{C}-\mathrm{V}$ model. As a consequence, it is clear that the present model can reduce to the generalized piezoelectric thermoelasticity model [16] if $\omega \longrightarrow 0$. The temperature responses of piezoelectric plate under transient thermal loadings are predicted and shown in Figure 2. It is shown that the results match well with [46].

As shown in Figures 3 and 4, two cases are analyzed and discussed: In Case 1, the solid lines represent the results from generalized piezoelectric thermoelasticity theory. In Case 2, the dashed lines are mainly devoted to evaluating the influence of time-delay and kernel function on the transient thermoelastopiezoelectric responses. For each problem, the computations are carried out at the instant $t=0.08$. Different values of time-delay are selected as $\omega=0.01$ and $\omega=0.05$. The kernel functions can be chosen freely as $K(t, p)=1$ and $K(t, p)=[(p-t) /(\omega+1)]$ [38]. The distributions of dimensionless temperature, displacement, stress, and electric potential are illustrated graphically. Problem 1 and Problem 2 are, respectively, plotted in Figures 3 and 4 .

It can be analytically obtained that the position of thermal wave front is at $x=0.35$ due to the fact that the velocity of thermal wave is $v_{\theta}=\sqrt{1 / \tau}=4.47$ (see equation (30)). It can be clearly seen from Figures 3(a) and Figure 4(a) that the evaluated thermal wave is closely approaching the theoretical analysis. Thus, the results are reliable by using Laplace transformation method. The distributions of dimensionless temperature are shown in Figures 3(a) and 4(a). 
TABLE 1: Material constants of cadmium selenide.

\begin{tabular}{lcr}
\hline$c_{33}=8.36 \times 10^{10} \mathrm{Nm}^{-2}$ & $\beta_{3}=0.55 \times 10^{6} \mathrm{Nm}^{-2} \mathrm{~K}^{-1}$ & $\rho=7.6 \times 10^{3} \mathrm{kgm}^{-3}$ \\
$c_{E}=420 \mathrm{Jkg}^{-1} \mathrm{~K}^{-1}$ & $e_{33}=0.347 \mathrm{Cm}^{-2}$ & $d_{3}=9.03 \times 10^{-11} \mathrm{~N}^{-1} \mathrm{~m}^{-2}$ \\
$p_{3}=-2.94 \times 10^{-6} \mathrm{Cm}^{-2} \mathrm{~K}^{-1}$ & $\kappa_{3}=1.4 \mathrm{Wm}^{-1} \mathrm{~K}^{-1}$ & $T_{0}=293 \mathrm{~K}$
\end{tabular}

$\tau=0.05$

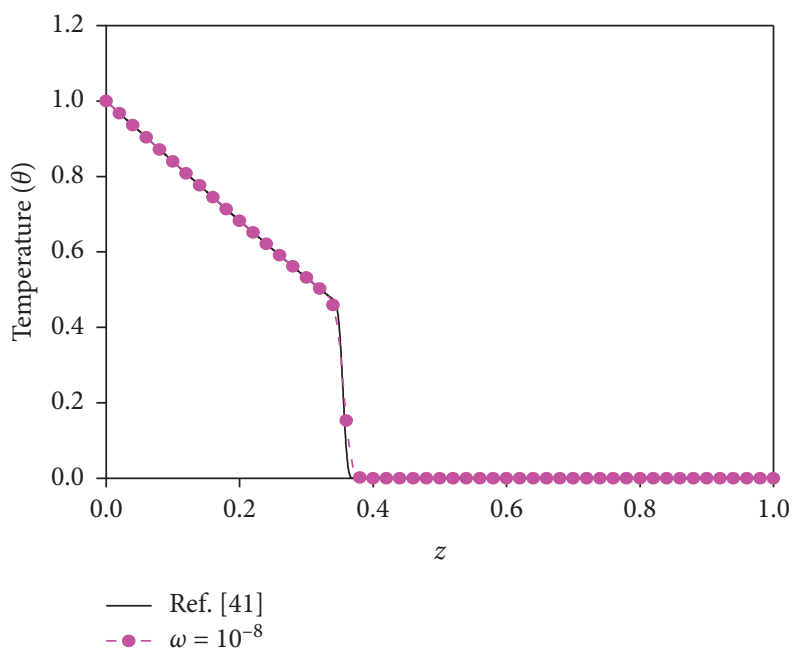

Figure 2: Comparison of the study of the present model and [41].

As seen from these figures, it can be summarized that timedelay has significant effect on the temperature: the distributions are smoother with time-delay increasing; the thermal wave front is dynamically smoothed and changed with the variations of time-delay and kernel function; the larger the time-delay, the faster the thermal wave travels.

The predicted dimensionless displacement distributions are, respectively, represented in Figures 3(b) and 4(b), which indicate that displacement's distributions are less affected by time-delay. As shown in Figures 3(c) and 4(c), it can be deduced that the distributions of the dimensionless stress around the thermal wave front are significantly affected by time-delay: the distributions are smoother when the timedelay is larger. As observed from Figures 3(d) and 4(d), it is noted that the distributions of dimensionless electric potential are greatly influenced by time-delay: with time-delay increasing, the distributions are smoother. Thus, we may arrive at a conclusion that the changes of displacement/ stress/electric potential are highly consistent with the influence of time-delay on temperature; that is, all the distributions of the considered field quantities are smoothed with time-delay increasing. The numerical results show the close interplay in thermoelectromechanical systems: when piezoelectric medium suffered from heating loads, an electric field will be stimulated due to piezoelectric effect, which reflects the thermoelectromechanical coupling effects.

Because the kernel function can be freely chosen, various transient thermoelastopiezoelectric responses can be captured (see Figures 3 and 4). More importantly, it can be found that the distributions of temperature, displacement, stress, and electric potential vanish identically outside a bounded region, which depends on the selections of timedelay and kernel function. Meanwhile, in the limit case of generalized piezoelectric thermoelasticity theory [16], the bounded region is merely time-related; that is, the location of thermal wave front is just dynamically changed with time. The numerical results indicate that the transient responses to thermal and piezoelectric effects do not reach infinity instantaneously; that is, the thermal wave and elastic wave propagate at a finite velocity.

To evaluate the effect of time on the structural dynamic responses, Figure 5 is graphically illustrated for Problem 1. Clearly, with the passage of time, the values of temperature, displacement, stress, and electric potential increase. Additionally, thermal wave will travel faster and the distributions of all the dynamic responses will become smoother. 

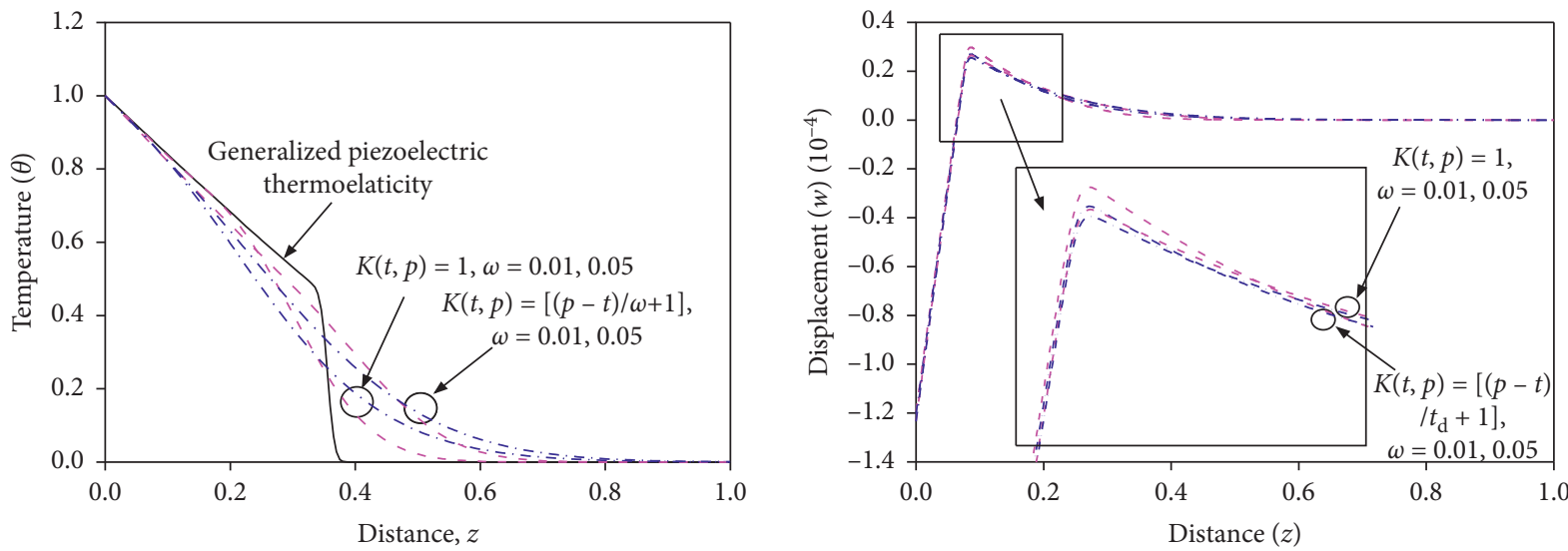

$$
\begin{aligned}
& -\omega=10^{-10} \\
& ---\omega=0.01 \\
& -. . \omega=0.05
\end{aligned}
$$$$
--\omega=0.01
$$$$
\text { -. . } \omega=0.05
$$

(a)

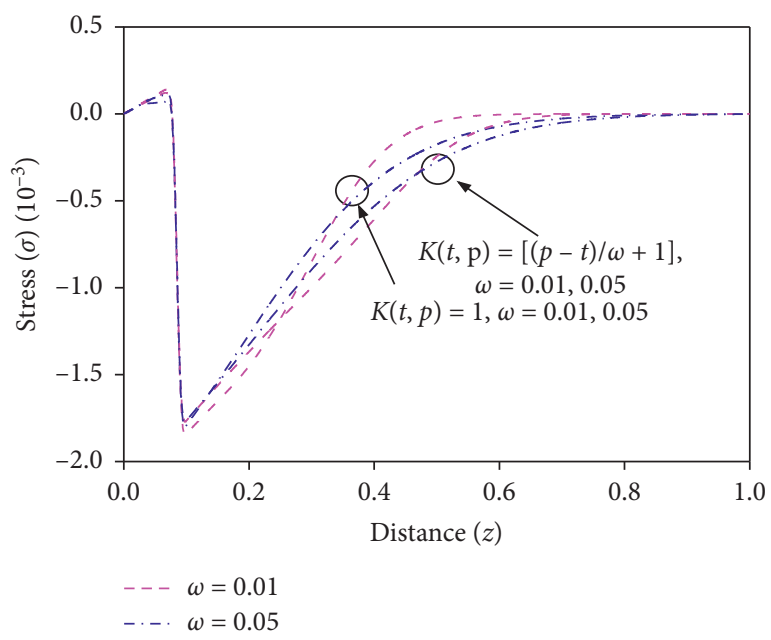

(c)

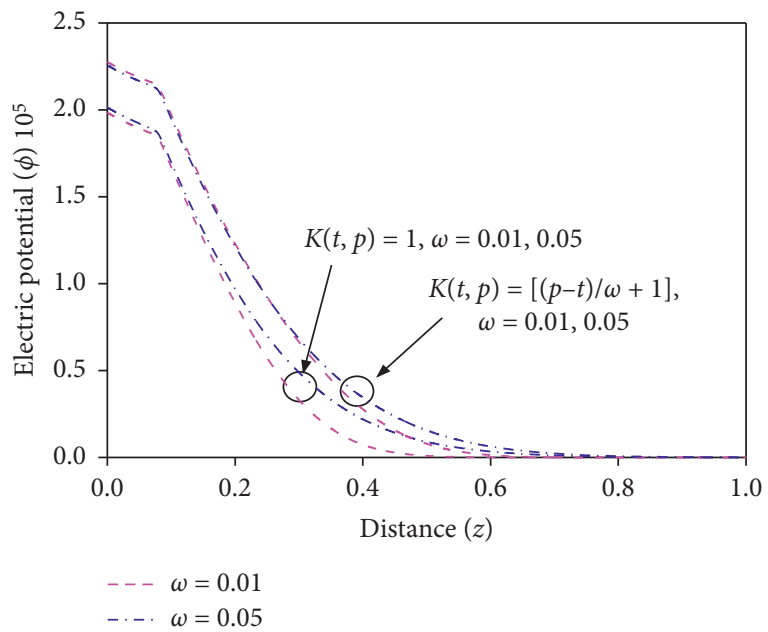

(d)

Figure 3: Distributions of temperature (a), displacement (b), stress (c), and electric potential (d) for different values of time-delay and kernel function in Problem 1.
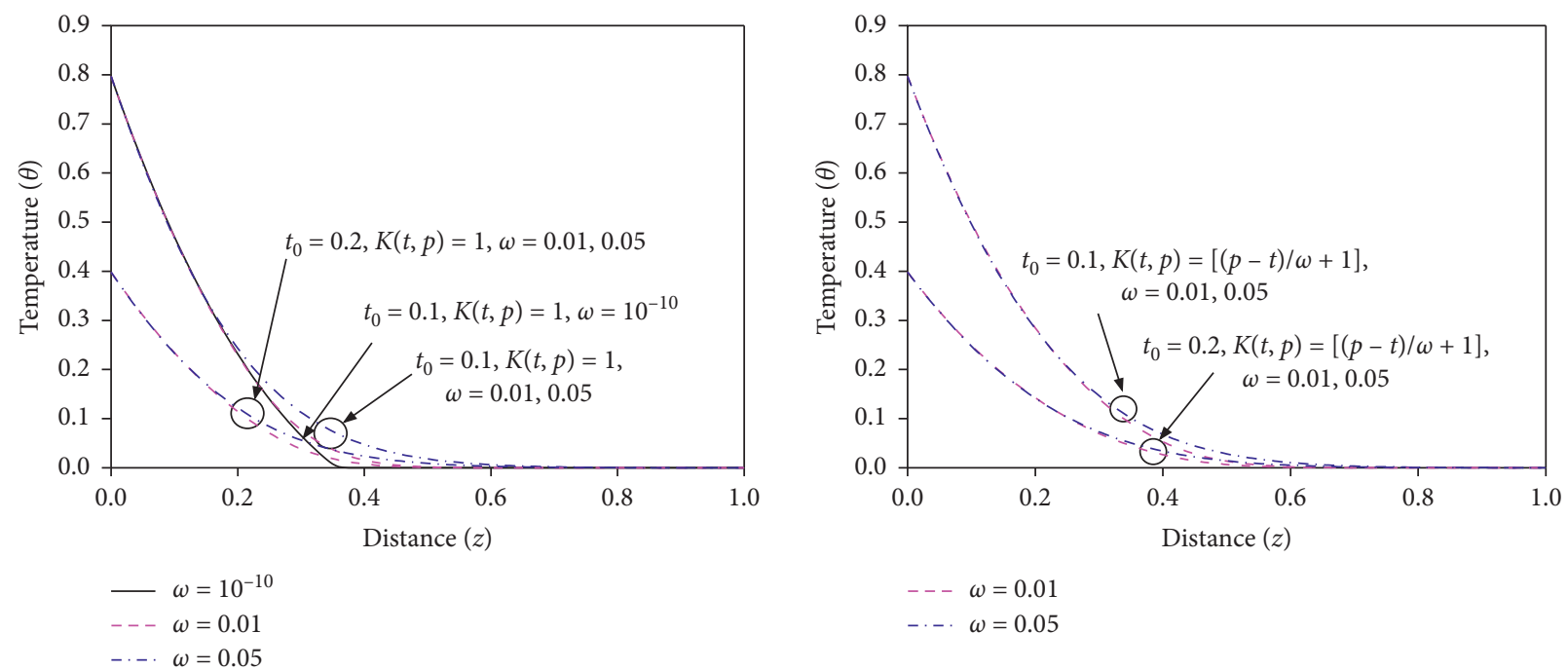

(a)

Figure 4: Continued. 

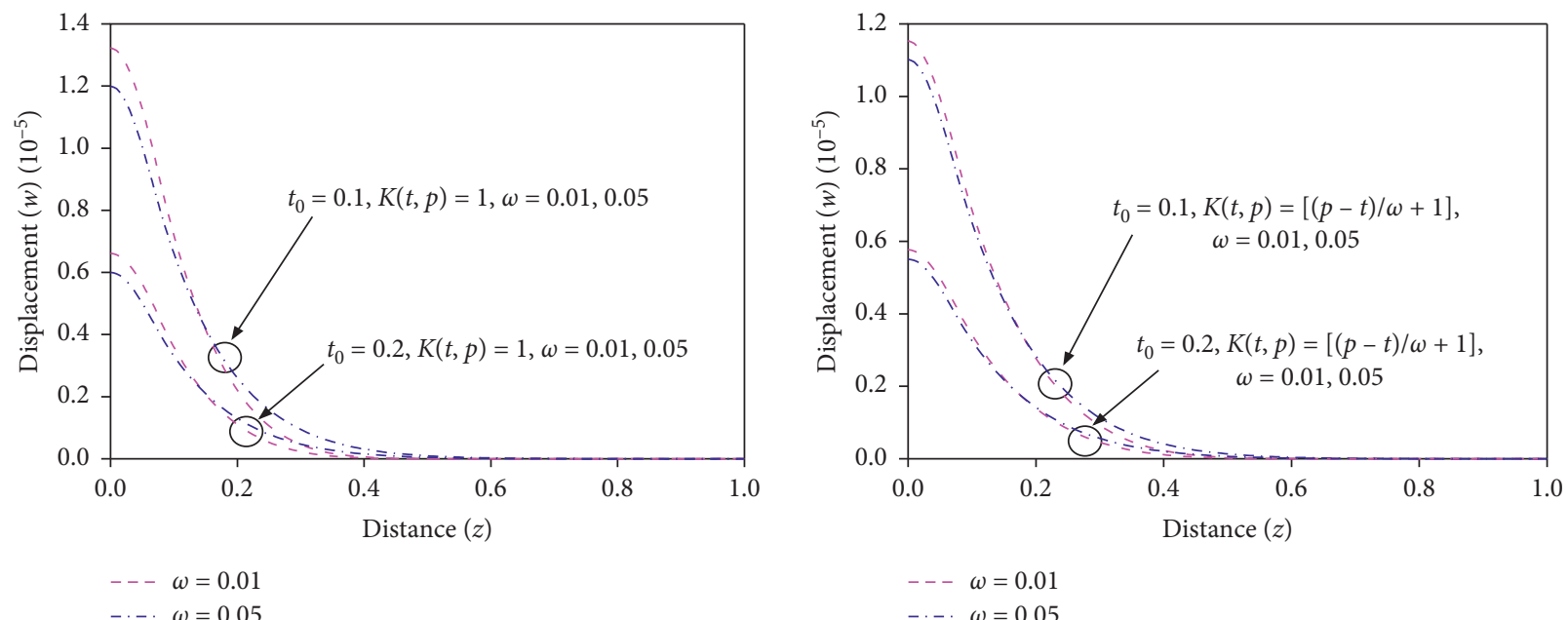

$---\omega=0.01$

-.. $\omega=0.05$

(b)
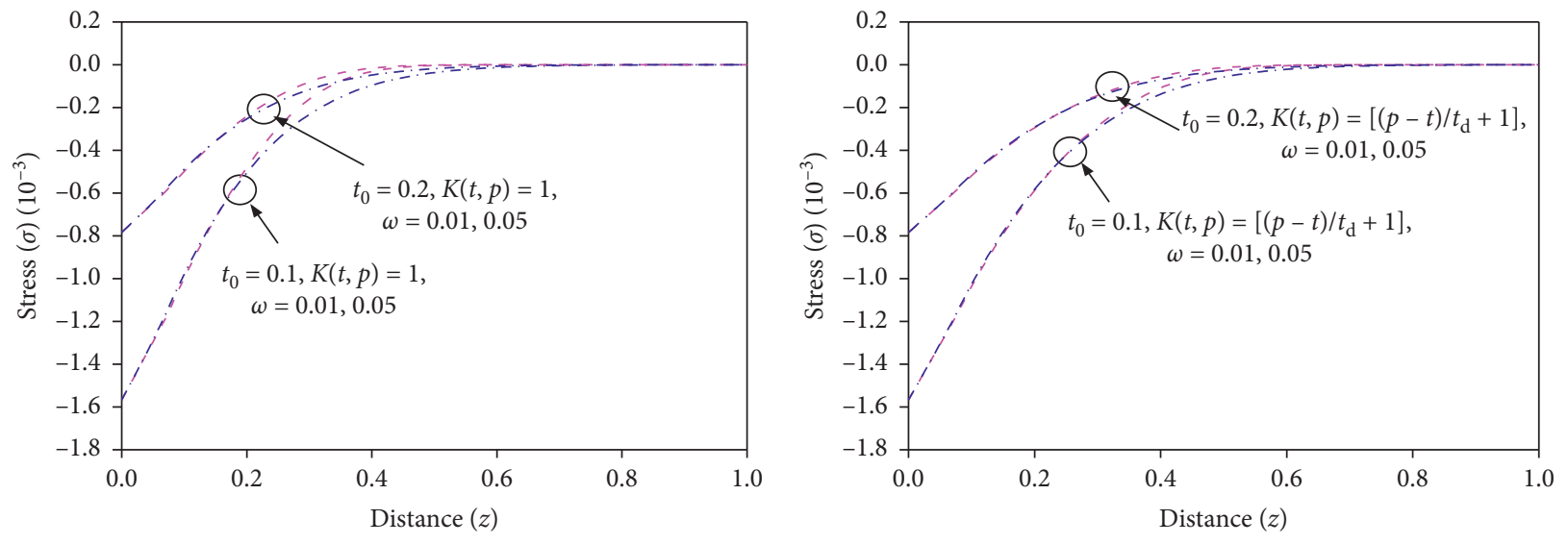

$---\omega=0.01$

$---\omega=0.01$

-. $\omega=0.05$

-.. $\omega=0.05$

(c)
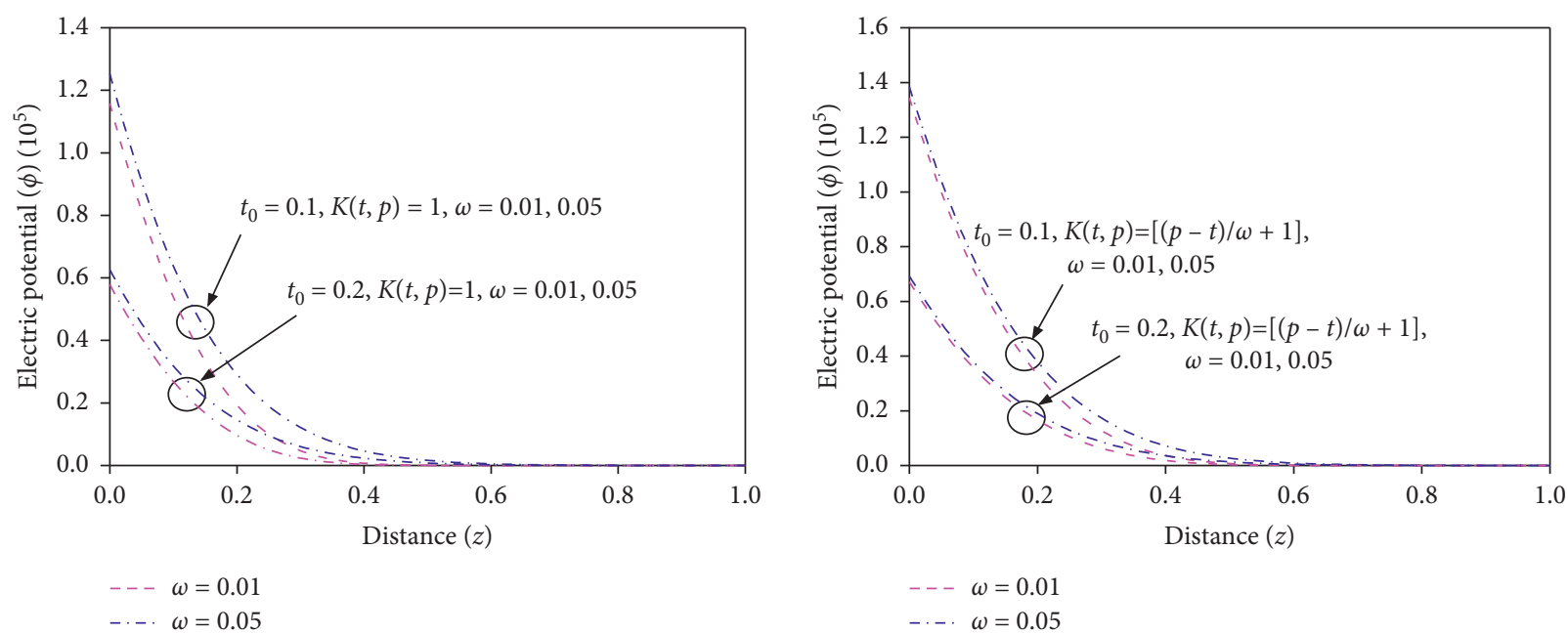

(d)

Figure 4: Distributions of temperature (a), displacement (b), stress (c), and electric potential (d) for different values of time-delay and kernel function in Problem 2. 


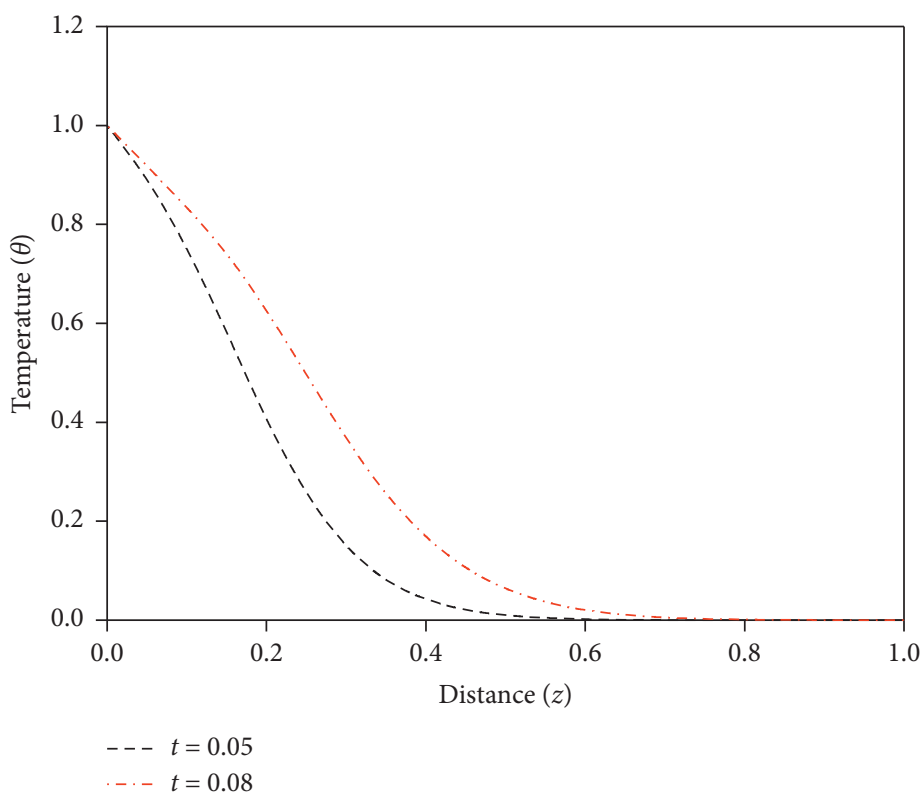

(a)

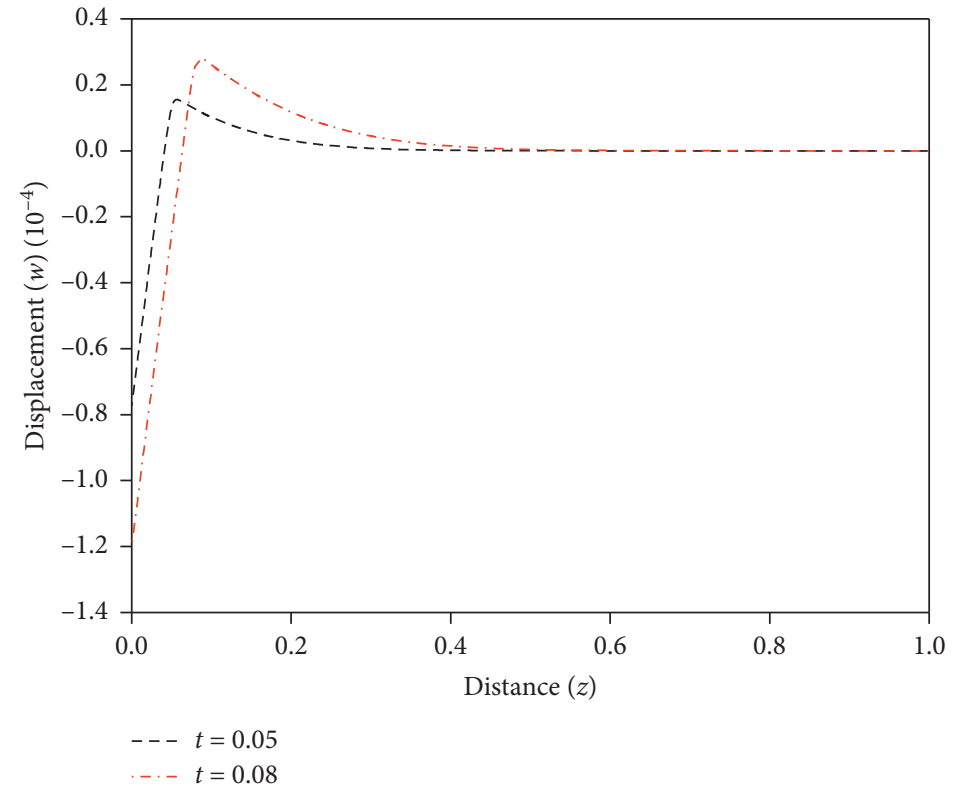

(b)

Figure 5: Continued. 


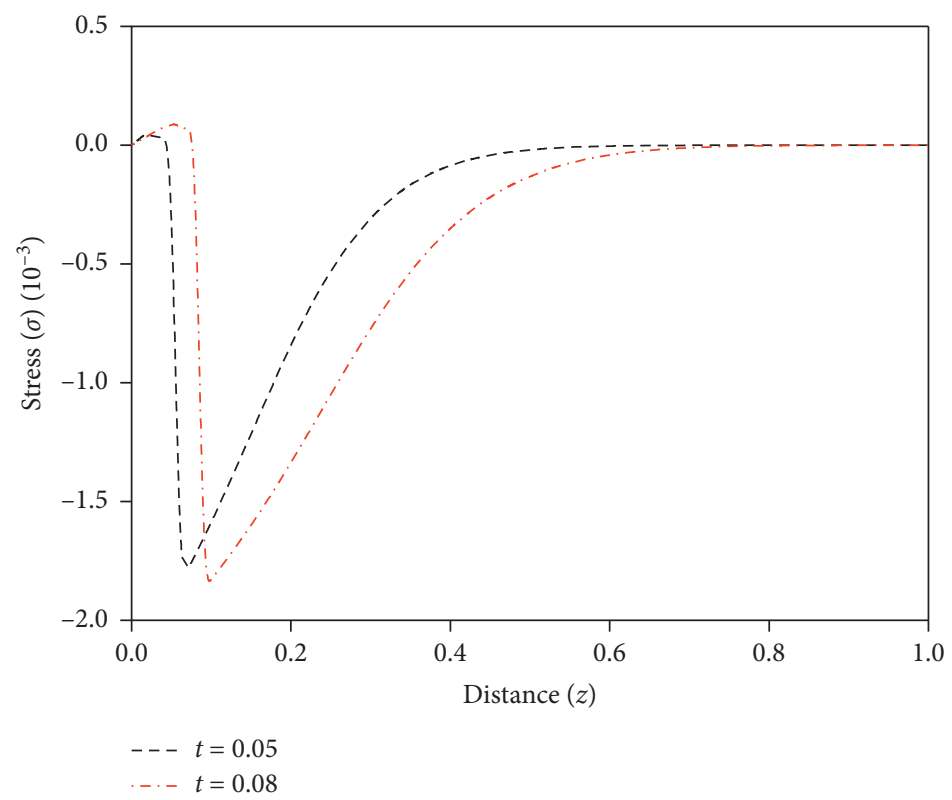

(c)

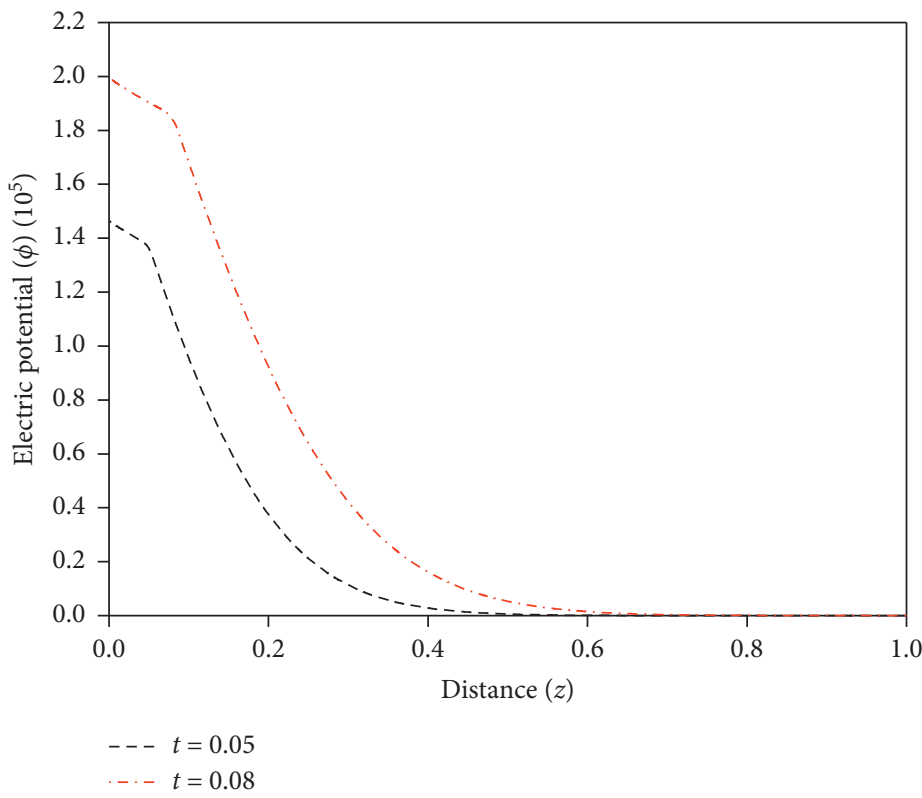

(d)

Figure 5: Distributions of temperature (a), displacement (b), stress (c), and electric potential (d) for different values of time in Problem 1.

The results show the excellent flexibility of the memorydependent derivative when investigating the micro-/nanoscale heating problem for piezoelectric medium.

\section{Conclusion}

This paper is mainly devoted to establishing a novel generalized thermopiezoelectricity by introducing memorydependent derivative and capturing the transient thermoelastopiezoelectric responses for a semi-infinite piezoelectric medium. The newly constructed model may be more rational and intuitionistic in reflecting the memory effect for piezoelectric medium under heating loads, which is intrinsically determined by the definition of memorydependent derivative. Laplace transformation techniques are employed to solve the governing equations. From the numerical results, the following conclusions can be summarized:

(1) It can be observed that the propagation of thermal wave is significantly affected by time-delay and kernel function: the thermal wave front is 
dynamically smoothed and changed with the introduction of memory-dependent derivative; the larger the time-delay is, the faster the thermal wave travels.

(2) Based on the necessity of applications, various transient responses can be captured by the flexible selections of time-delay and kernel function. Thus, it provides more approaches to describe piezoelectric material's responses' behavior in different processes.

(3) The distributions reveal the memory effect in the transient responses. In addition, it can be seen that the distributions of all field quantities are smoother when the time-delay is increasing.

(4) It is clear that, for small values of time, the bounded region of nonzero values for all the considered variables is dependent on the values of time-delay and the forms of kernel function.

(5) The parameter time-delay can be viewed as a new indicator when investigating the responses' behavior for piezoelectric medium under transient (even micro-/nanoscale) heating loads.

\section{Data Availability}

The data used to support the findings of this study are included within the article.

\section{Conflicts of Interest}

The authors declare that they have no conflicts of interest.

\section{Acknowledgments}

This work was supported by the National Natural Science Foundation of China (51236003).

\section{References}

[1] S. S. Rao and M. Sunar, "Piezoelectricity and its use in disturbance sensing and control of flexible structures: a survey," Applied Mechanics Reviews, vol. 47, no. 4, pp. 113-123, 1994.

[2] V. Z. Parton and B. A. Kudryavtsev, Electromagnetoelasticity, Gordon and Breach, New York, NY, USA, 1988.

[3] C. Li, H. Guo, X. Tian, and T. He, "Size-dependent thermoelectromechanical responses analysis of multi-layered piezoelectric nanoplates for vibration control," Composite Structures, vol. 225, Article ID 111112, 2019.

[4] C. L. Li, H. L. Guo, X. G. Tian, and T. H. He, "Generalized piezoelectric thermoelasticity problems with strain rate and transient thermo-electromechanical responses analysis," ZAMM-Z angew math me, vol. 100, Article ID e201900067, 2020.

[5] R. D. Mindlin, "Equations of high frequency vibrations of thermopiezoelectric crystal plates," International Journal of Solids and Structures, vol. 10, no. 6, pp. 625-637, 1974.

[6] W. Nowacki, "Some general theorems of thermopiezoelectricity," Journal of Thermal Stresses, vol. 1, no. 2, pp. 171-182, 1978.

[7] Y. Ootao, T. Akai, and Y. Tanigawa, "Transient piezothermoelastic analysis for a functionally graded thermopiezoelectric hollow cylinder," Journal of Thermal Stresses, vol. 31, no. 10, pp. 935-955, 2008.

[8] V. Gupta, M. Sharma, N. Thakur, and S. P. Singh, "Active vibration control of a smart plate using a piezoelectric sensoractuator pair at elevated temperatures," Smart Materials and Structures, vol. 20, no. 10, Article ID 105023, 2011.

[9] A. B. Zhang and B. L. Wang, "Crack branching in thermopiezoelectric materials," International Journal of Solids and Structures, vol. 50, no. 19, pp. 2962-2969, 2013.

[10] A. K. Vashishth, H. Sukhija, and V. Gupta, "Ultrasonic waves in an orthorhombic porous piezo-thermoelastic laminated structure immersed in a fluid," Smart Materials and Structures, vol. 25, no. 11, Article ID 115025, 2016.

[11] V. Peshkov, "Second sound in helium II," Journal of Physics, vol. 8, pp. 381-382, 1944.

[12] C. C. Ackerman, B. Bertman, H. A. Fairbank, and R. A. Guyer, "Second sound in solid helium," Physical Review Letters, vol. 16, no. 18, pp. 789-791, 1966.

[13] C. C. Ackerman and W. C. Overton, "Second sound in solid helium-3," Physical Review Letters, vol. 22, no. 15, pp. 764-766, 1969.

[14] C. Cattaneo, "A form of heat equation which eliminates the paradox of instantaneous propagation," Comptes Rendus de l'Académie des Sciences, vol. 247, pp. 431-433, 1958.

[15] P. Vernotte, "Paradoxes in the continuous theory of the heat conduction," Comptes Rendus de l'Académie des Sciences, vol. 246, pp. 3154-3155, 1958.

[16] D. S. Chandrasekharaiah, "A generalized linear thermoelasticity theory for piezoelectric media," Acta Mechanica, vol. 71, pp. 39-49, 1998.

[17] T. H. He, X. G. Tian, and Y. P. Shen, "Two-dimensional generalized thermal shock problem of a thick piezoelectric plate of infinite extent," International Journal of Engineering Science, vol. 40, pp. 2249-2264, 2002.

[18] X. Tian, J. Zhang, Y. Shen, and T. J. Lu, "Finite element method for generalized piezothermoelastic problems," International Journal of Solids and Structures, vol. 44, no. 18-19, pp. 6330-6339, 2007.

[19] M. Aouadi, "Generalized thermo-piezoelectric problems with temperature-dependent properties," International Journal of Solids and Structures, vol. 43, no. 21, pp. 6347-6358, 2006.

[20] M. H. Babaei and Z. T. Chen, "Dynamic response of a thermopiezoelectric rod due to a moving heat source," Smart Materials and Structures, vol. 18, pp. 1-9, 2009.

[21] M. H. Babaei and Z. T. Chen, "Transient thermopiezoelectric response of a one-dimensional functionally graded piezoelectric medium to a moving heat source," Archive of Applied Mechanics, vol. 80, no. 7, pp. 803-813, 2010.

[22] A.-e.-n. N. Abd-Alla, A. M. Hamdan, I. Giorgio, and D. Del Vescovo, "The mathematical model of reflection and refraction of longitudinal waves in thermo-piezoelectric materials," Archive of Applied Mechanics, vol. 84, no. 9-11, pp. 1229-1248, 2014.

[23] T. Q. Qiu and C. L. Tien, "Short-pulse laser heating on metals," International Journal of Heat and Mass Transfer, vol. 35, no. 3, pp. 719-726, 1992.

[24] D. Y. Tzou, J. K. Chen, and J. E. Beraun, "Hot-electron blast induced by ultrashort-pulsed lasers in layered media," International Journal of Heat and Mass Transfer, vol. 45, no. 16, pp. 3369-3382, 2002.

[25] T. Q. Qiu and C. L. Tien, "Femtosecond laser heating of multilayer metals-I. Analysis," International Journal of Heat and Mass Transfer, vol. 37, no. 17, pp. 2789-2797, 1994. 
[26] J. Peddieson, G. R. Buchanan, and R. P. McNitt, “Application of nonlocal continuum models to nanotechnology," International Journal of Engineering Science, vol. 41, no. 3-5, pp. 305-312, 2003.

[27] D. D. Joseph and L. Preziosi, "Heat waves," Reviews of Modern Physics, vol. 61, no. 1, pp. 41-73, 1989.

[28] A. Kullberg, G. J. Morales, and J. E. Maggs, "Comparison of a radial fractional transport model with tokamak experiments," Physics of Plasmas, vol. 21, Article ID 032310, 2015.

[29] D. Del-Castillo-Negrede, P. Mantica, V. Naulin, and J. J. Rasmussen, "Fractional diffusion models of non-local perturbative transport: numerical results and application to JET experiments," Nuclear Fusion, vol. 48, Article ID 075009, 2008.

[30] Y. Z. Povstenko, "Fractional heat conduction equation and associated thermal stress," J. Journal of Thermal Stresses, vol. 28, pp. 83-102, 2005.

[31] Y. Z. Povstenko, "Fractional radial heat conduction in an infinite medium with a cylindrical cavity and associated thermal stresses," Mechanics Research Communications, vol. 37, no. 4, pp. 436-440, 2010.

[32] T. Atanackovic, S. Konjik, L. Oparnica, and D. Zorica, "The Cattaneo type space-time fractional heat conduction equation," Continuum Mechanics and Thermodynamics, vol. 24, pp. 293-311, 2012.

[33] H. Qi and X. Guo, "Transient fractional heat conduction with generalized Cattaneo model," International Journal of Heat and Mass Transfer, vol. 76, pp. 535-539, 2014.

[34] A. E. Abouelregal, "Fractional order generalized thermopiezoelectric semi-infinite medium with temperaturedependent properties subjected to a ramp-type heating," Journal of Thermal Stresses, vol. 34, no. 11, pp. 1139-1155, 2011.

[35] M. Islam and M. Kanoria, "One-dimensional problem of a fractional order two-temperature generalized thermo-piezoelasticity," Mathematics and Mechanics of Solids, vol. 19, no. 6, pp. 672-693, 2014.

[36] Y. B. Ma and T. H. He, "Dynamic response of a generalized piezoelectric-thermoelastic problem under fractional order theory thermoelasticity," Mechanics of Advanced Materials and Structures, vol. 23, pp. 1173-1180, 2015.

[37] I. Podlubny, Fractional Differential Equations, Academic, New York, NY, USA, 1999.

[38] J.-L. Wang and H.-F. Li, "Surpassing the fractional derivative: concept of the memory-dependent derivative," Computers \& Mathematics with Applications, vol. 62, no. 3, pp. 1562-1567, 2011.

[39] A. Sur, "The memory effect on thermal wave propagation in a moving thin slab," Waves in Random and Complex Media, vol. $1,2020$.

[40] A. Sur and S. Mondal, "The Caputo-Fabrizio heat transport law in vibration analysis of a microscale beam induced by laser," ZAMM-Journal of Applied Mathematics and Mechanics/Zeitschrift für Angewandte Mathematik und Mecha$n i k$, vol. 10, 2020.

[41] A. Sur, "Memory response on wave propagation in a micropolar magneto-thermo-viscoelastic half-space," Waves in Random and Complex Media, vol. 10, 2020.

[42] A. Sur and M. I. A. Othman, "Elasto-thermodiffusive interaction subjected to rectangular thermal pulse and timedependent chemical shock due to caputo-fabrizio heat transfer," Waves in Random and Complex Media, vol. 11 page, 2020 .
[43] A. Sur, S. Mondal, and M. Kanoria, "Effect of hydrostatic pressure and memory effect on magneto-thermoelastic materials with two-temperatures," Waves in Random and Complex Media, vol. 1, 2020.

[44] L. Brancik, "Programs for fast numerical inversion of Laplace transforms in MATLAB language environment," in Proceedings of the Seventh Prague Conference MATLAB'99, pp. 27-39, Stresa, Italy, August 1999.

[45] T. H. He and Y. Li, "Transient responses of sandwich structure based on the generalized thermoelastic diffusion theory with memory-dependent derivative," Journal of Sandwich Structures \& Materials, vol. 1-39, 2018.

[46] C. Li, H. Guo, and X. Tian, "Size-dependent effect on thermoelectro-mechanical responses of heated nano-sized piezoelectric plate," Waves in Random and Complex Media, vol. 29, no. 3, pp. 477-495, 2019. 